SOFIA

\title{
A Stratospheric Observatory for Infrared Astronomy
}

H.-P. Röser and G. Mezger of the Max-Planck-Institut für Radioastronomie, Bonn and members of the SOFIA Science Working Group report on progress

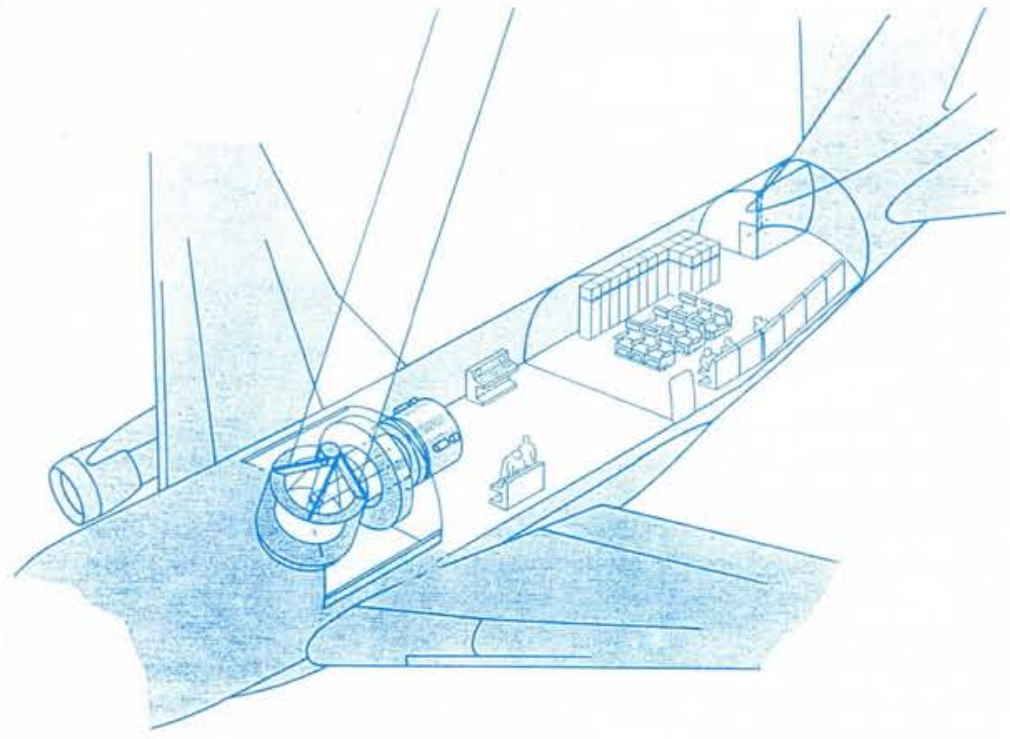

SOFIA stratospheric observatory for IR astronomy: illustration of the 2,7 $m$ telescope installed in the Boeing 747 aircraft.

SOFIA is an airborne observatory designed to address fundamental questions relating to galactic and extragalactic astronomy, and to the origin and evolution of the solar system. Much of the radiant energy in the Universe lies at infrared wavelengths which are not observable using ground-based telescopes. SOFIA will provide ready and frequent access to these wavelengths with spatial and spectral resolutions that will not be matched this century.

SOFIA comprises a telescope, with an overall diameter of $2.7 \mathrm{~m}$ and an effective aperture diameter (entrance pupil) of 2.5 $\mathrm{m}$, operating from a Boeing 747 aircraft at altitudes between 41000 and 45000 feet. It will provide astronomers with routine access to infrared, far infrared and submillimetric wavelengths unavailable on the ground, and with the means to observe transient astronomical events from anywhere in the world.

The project is currently being studied jointly by NASA and the German Science Ministry (BMFT), Phase B studies on aircraft modification and the telescope system having been completed in 1989 (Phases C and D should start at the end of 1990). The overall concept is based on 15 years of experience with NASA's Kuiper Airborne Observatory (KAO) which SOFIA will replace in the mid 1990's. However, SOFIA's wavelength range covers nearly four decades of the electromagnetic spectrum, from the visible, through the infrared and submillimetric, to the microwave region. It will thus be roughly ten times more sensitive for compact sources than $\mathrm{KAO}$, enabling observations of fainter objects and measurements at a higher spectral resolution. The angular resolving power for wavelengths above 30 microns will be three times larger, permitting more detailed imaging in the far infrared.

\section{Scientific Objectives}

The spectral regime from the infrared to the submillimetre encompasses a multitude of rich and varied physical processes, and is uniquely suited for studying the cosmic birth on all scales. SOFIA's high spectral and spatial resolutions will exploit and extend the scientific legacy left by the IRAS (Infrared Astronomical Satellite), and will complement future space observatories such as ISO (Infrared Space Observatory), SIRTF (Space Infrared Telescope Facility) and FIRST (Far Infrared Space Telescope).

Questions that would be addressed by SOFIA are described in the box on page 119.

\section{Atmospheric Physics}

Most of the atoms and molecules which are relevant for astronomy are also of interest for atmospheric physics. Using the SOFIA telescope with or without additional transparent windows (up-, downand sidewards looking, e.g. in the rear of the aircraft) the observatory could contribute significantly to atmospheric physics. The flying laboratory would make it possible to investigate, for instance, global and local concentrations of molecules at different altitudes virtually anywhere in the world.

\section{The Programme}

SOFIA will be operated by NASA with support from the BMFT as an international facility for astronomy throughout a 20 year lifetime. It will provide 120 eight hour flights each year for approximately 40 principal investigation teams selected annually by peer review.

Roughly half of the teams will provide the observatory with a wide variety of specialized instruments, including arrays of cameras, polarimeters, and several types of spectrometers. State-of-the-art focal plane instrumentation is a key aspect of the SOFIA concept so, in addition to its wide-ranging scientific contributions, SOFIA will stimulate development of focal plane instrument technologies. The SOFIA programme will also extensively help the scientific community to support future astronomic missions.

\section{The Observatory}

The stratospheric observatory will be a joint project, under NASA leadership, between the US and the Federal Republic of Germany, which has tentatively agreed to provide a significant contribution to the development by supplying a major part of the telescope system (in addition to operating costs commensurate with the fraction of flight time the German science community would receive). It is expected that the Federal Republic will assume a share of about $20 \%$ of the costs needed to construct and operate the airborne facility. A collaboration agreement would ensure that German scientific teams are guaranteed $20 \%$ of the observing time (approximately 25 flights). A Memorandum of Understanding to this effect was signed by both sides in 1990 .

Teams from other countries are being invited to negotiate for time with the US proposers on the same basis as for KAO. The USA and the Federal Republic have also agreed that other European countries could participate in the German technical and financial contribution to SOFIA in exchange for an equivalent fraction of the flight time. Italy and The Netherlands started to formalise such an arrangement at the beginning of the year.

\section{FURTHER INFORMATION}

[1] The SOFIA Science Working Group is chaired by E.F. Erickson, NASA Ames Research Center, Moffett Field, CA 94035, USA.

[2] Phase A and B System Concept Descriptions: available from the authors upon request. Address:

Max-Planck-Institut für Radioastronomie, Auf dem Hügel 69, D-5300 Bonn 1, FRG. Tel.: ++49 (228) $525392 /-297$

Fax: ++49 (228) 525229

[3] Röser H.P. and Schmid-Burgk J., Sterne und Weltraum 28 (1989) 648. 